\title{
Análisis de Esquemas de Metadatos para la Marcación de Contenidos Educativos
}

\author{
Angela M. Vargas-Arcila(1), Sandra Baldassarri ${ }^{(2)}$ y José L. Arciniegas ${ }^{(1)}$ \\ (1) Universidad del Cauca, CII 5 \# 4-70, Popayán-Colombia. (e-mail: amvargas@unicauca.edu.co, \\ jlarci@unicauca.edu.co) \\ (2) Universidad de Zaragoza, María de Luna 1, 50018 Zaragoza-España. (e-mail: sandra@unizar.es)
}

Recibido Dic. 23, 2015; Aceptado Feb. 22, 2016; Versión final Mar. 31, 2016, Publicado Oct. 2016

\begin{abstract}
Resumen
Este trabajo presenta un análisis de los esquemas de metadatos más relevantes que se han encontrado en la literatura para la marcación de contenido educativo en la Web: LOM, MLR, LRMI, DC-Ed AP y EdNA). El resultado de este análisis se refleja en un gráfico de relación entre los esquemas y en una tabla comparativa de las características más importantes de cada uno de ellos. LOM es el estándar de metadatos con mayor repercusión en e-learning y continúa vigente. Sin embargo, la gran cantidad de perfiles de aplicación que se han implementado a partir de él evidencian su incapacidad de soportar la necesidades de diferentes contextos educativos. Se encuentra también que MLR y LRMI son estándares actuales que introducen elementos pedagógicos ausentes en esquemas más antiguos.
\end{abstract}

Palabras clave: esquema de metadatos, contenidos educativos, LOM, MLR, LRMI, DC-Ed AP, EdNA.

\section{Analysis of Metadata Schemas for Marking Up Educational Content}

\begin{abstract}
This paper presents an analysis of the most relevant metadata schemas for marking up educational content found on the Web: LOM, MLR, LRMI, DC-Ed AP and EdNA. The results of this analysis are summarized in a plot that relates the different schemes and in a table in which the main characteristic are compared. LOM is the standard of metadata with the highest impact on e-learning and continue being used. However, the great amount of application profiles that have been implemented, gives an indication about its incapacity of supporting the needs of different educational contexts. It is also found that MLR and LRMI are the current standards that introduce educational elements absent in older schemas.
\end{abstract}




\section{INTRODUCCIÓN}

Los procesos de formación actuales incorporan, cada vez más, tecnologías que facilitan el aprendizaje. Dichos procesos se pueden denominar por medio de un término ya establecido que depende de la medida y manera en que son utilizadas las tecnologías. Habitualmente el proceso de enseñanza aprendizaje soportado por las tecnologías de la información, especialmente Internet, es denominado e-learning (Morales, 2007), sin embargo, éste es un término mucho más amplio que no necesariamente se refiere a una modalidad de formación a distancia, sino que también se utiliza en procesos de aprendizaje que combinan situaciones presenciales y no presenciales, convirtiéndose en lo que se denomina blendedlearning o b-learning; por otro lado, si el proceso de aprendizaje se lleva a cabo en cualquier momento y lugar por medio de dispositivos móviles (teléfono móvil, tableta, computador portátil, etc.), también se puede denominar m-learning. Teniendo en cuenta la diversidad de elementos que abarcan los diferentes tipos de formación, es necesario garantizar la interoperabilidad, reusabilidad y portabilidad de los recursos educativos utilizados en cada una de las plataformas (Friesen, 2004). Para ello es importante que los contenidos educativos sean descritos e identificados adecuadamente por medio de un conjunto de metadatos bien definidos.

Los metadatos son información que describe, identifica, explica o define un recurso con el objetivo de facilitar su recuperación, uso o gestión (NISO, 2004). Generalmente se utilizan para referirse a registros descriptivos de recursos digitales que sirven para resumir el contenido del recurso, permitir su búsqueda o recuperación, especificar características de propiedad, proporcionar información acerca de cómo interpretarlo, detallar sus condiciones de uso, y especificar las relaciones con otros recursos, etc. (Frederick, 2016). En el contexto educativo, un metadato es información acerca de un recurso educativo, que permite describir diferentes aspectos acerca de su contenido y sus objetivos, facilitando su búsqueda, selección y recuperación (Fernández-Manjón et al, 2011). Los conjuntos de metadatos relacionados con un recurso, tienen una estructura bien definida y un conjunto de reglas para su uso, denominada esquema de metadatos. Un esquema de metadatos, a su vez, puede ser modificado para una situación específica de dos maneras: por medio de la creación de un perfil de aplicación, es decir, la combinación de metadatos de diferentes esquemas o la limitación y refinamiento de los existentes, y por medio de la creación de una extensión del esquema, es decir, adición de nuevos metadatos. Existen diversos esquemas de metadatos para la marcación de recursos educativos los cuales han sido recopilados en diferentes trabajos como (Berlanga y García, 2004) (Ponds et al, 2011) (Fernández-Manjón et al, 2011) (Castro-García y LópezMorteo, 2013) (Nevile, 2013), sin embargo, no se ha encontrado en la literatura un estudio actual que realice una comparación de las características de los esquemas más destacados e ilustre las relaciones existentes entre ellos. Por este motivo en este artículo se seleccionan los esquemas de metadatos más relevantes para la marcación de recursos educativos en la web, con el objetivo de aclarar sus relaciones y características.

\section{ESQUEMAS DE METADATOS}

Actualmente, la educación se ve cada vez más influenciada por las tecnologías de la información y las comunicaciones (TIC) de diferentes maneras, en especial, Internet es el elemento que ha cambiado, entre muchas cosas, la forma de trabajar, los métodos de comunicación y la manera de interactuar directamente con otras personas, por ende, es capaz de respaldar la educación eliminando las barreras geográficas. En representación de lo anterior, se puede hacer referencia a los foros y grupos de discusión, cursos en línea, buscadores que permiten encontrar referencias que enriquecen el aprendizaje, o cualquier otra herramienta en línea que pueda apoyar un proceso formal o informal de formación. Generalmente, a este tipo de interacción educativa por medio de Internet se le denomina de diferentes maneras, tales como, teleformación, formación en línea, formación virtual, o e-learning (Fernández-Manjón et al, 2011).

En el proceso de enseñanza aprendizaje por medio de la web, suman a los estudiantes y profesores, los proveedores de contenido y los administradores. Los proveedores de contenido se encargan de crear y diseñar el contenido (documentos, imágenes, videos) de acuerdo a un objetivo educativo. Los administradores, por su parte, son actores que participan en el proceso cuando éste es soportado por un sistema de gestión de aprendizaje, su función es gestionar los recursos educativos, cursos, horarios, sesiones de aprendizaje, tutores, seguridad del sistema, etc. Es importante aclarar, que los actores del proceso no necesariamente son independientes, pues es habitual que el profesor, además de desempeñarse como dinamizador del proceso de enseñanza, también sea el principal proveedor de contenidos para dicho proceso (Fernández-Manjón et al, 2011).

Con el objetivo de reutilizar los recursos educativos usados en un proceso de enseñanza en línea, ha sido necesario establecer estándares que permitan la interoperabilidad de los elementos implicados en dicho proceso. El trabajo (Fernández-Manjón et al, 2011) identifica ocho capas en las que se deben definir estándares para lograr la interoperabilidad (ver fig. 1). 


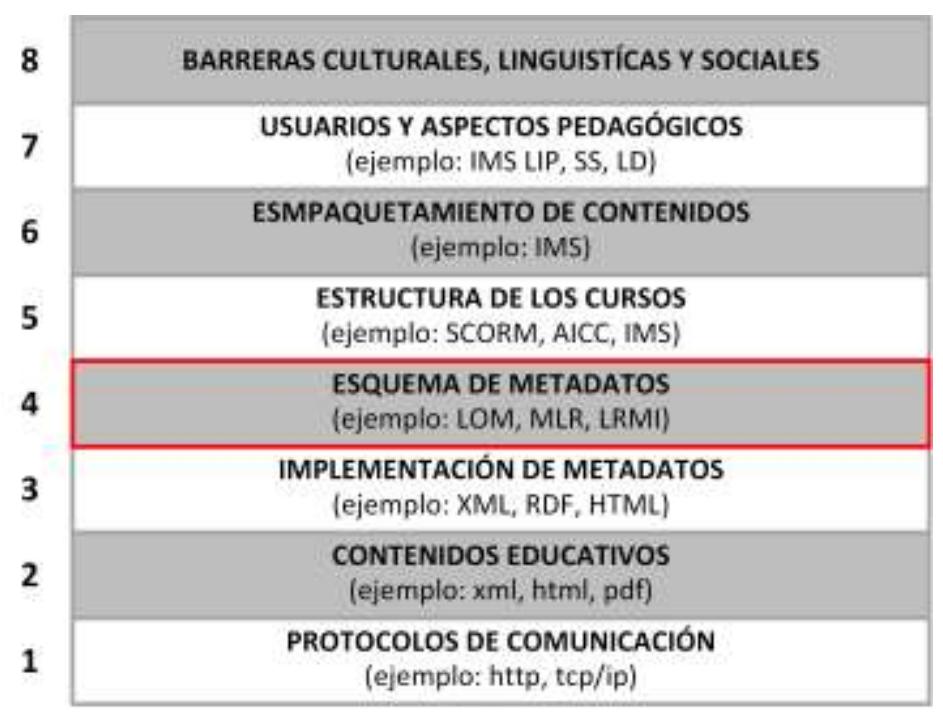

Fig. 1: Modelo en capas para llegar a la interoperabilidad de los recursos de e-learning (Fernández-Manjón et al, 2011).

La capa Protocolos de Comunicación, se refiere al conjunto de protocolos que permiten la comunicación en una red (por ejemplo: TCP/IP, HTTP, etc.). La capa Contenidos Educativos, hace referencia a los formatos en los que se crean los contenidos educativos y que son soportados por los navegadores (por ejemplo: xml, html, pdf, etc.). En la capa Implementación de Metadatos se encuentran los lenguajes que pueden ser utilizados para representar los metadatos relacionados con los contenidos educativos (por ejemplo: XML, RDF, HTML). La capa Esquema de Metadatos representa el conjunto de metadatos necesarios y la relación entre los mismos, para describir la información relevante relacionada con el contenido educativo. Las capas Estructura de los Cursos y Empaquetamiento de Contenidos hacen referencia a las maneras de relacionar y estructurar contenidos educativos para crear cursos y asegurar su portabilidad entre diferentes sistemas de aprendizaje. La capa Usuarios y Aspectos Pedagógicos caracterizan los perfiles de los actores implicados en el proceso de enseñanza aprendizaje y la manera de utilizar los recursos. Finalmente la capa Barreras Culturales, Lingüísticas y Sociales, contiene los elementos necesarios para adaptar los elementos educativos a diferentes contextos (idioma, cultura y sociedad) (Fernández-Manjón et al, 2011).

Este trabajo se centra en los esfuerzos relacionados con la capa 4, es decir, en los esquemas de metadatos educativos desarrollados por el momento, con el fin de encontrar una relación clara entre los mismos y comparar algunas de sus características principales. Se ha considerado diferentes esquemas de metadatos, extensiones y perfiles de aplicación que pueden ser utilizados para la marcación de recursos educativos. De todos los existentes, sin embargo, se han seleccionado y se describen los más recientes (Metadata for Learning Resources (MLR) y Learning Resource Metadata Initiative (LRMI) (Hoel, 2011) (Waters, 2013)), influyentes (IEEE Learning Object Metadata / IMS Learning Resource Metadata Specification (LOM)) (Castro-García, 2013) o vigentes (EdNA Metadata Standard y Dublin Core Education Application Profile (DC-Ed AP) (Barker, 2010)).

\section{EdNA Metadata Standard}

El estándar de metadatos EdNA (EdNA Metadata Standard) fue desarrollado por la Red de Educación de Australia (EdNA, Education Network Australia), la cual es un proyecto para la cooperación entre todos los sectores de la educación y formación australiana enfocados en las tecnologías de la información. Su propósito es respaldar la interoperabilidad entre dichos sectores en cuanto a descubrimiento y gestión de recursos en línea. Actualmente su principal aplicación es facilitar el almacenamiento de metadatos de recursos educativos de todos los estados y territorios de Australia. Su primera versión (1.0) fue publicada en 1998 y la versión vigente es la 1.1 que fue ratificada en el 2000 (EdNA, s.f).

De acuerdo a las decisiones del gobierno australiano y a los esfuerzos internacionales de metadatos, el Comité de Referencia EdNA (actualmente AICTEC, Australian Information and Communication Technologies in Education Committee) acordó que este estándar de metadatos se debía basar en el conjunto de metadatos de Dublin Core (DC), por esta razón, para EdNA son relevantes los principios importantes de DC, tales como, simplicidad, interoperabilidad y extensibilidad (EdNA, s.f). En consecuencia, EdNA conserva los quince elementos definidos por Dublin Core y agrega ocho nuevos metadatos, sin embargo, estos últimos no representan características educativas ni una relación del recurso con un proceso educativo (ver fig. 2). 


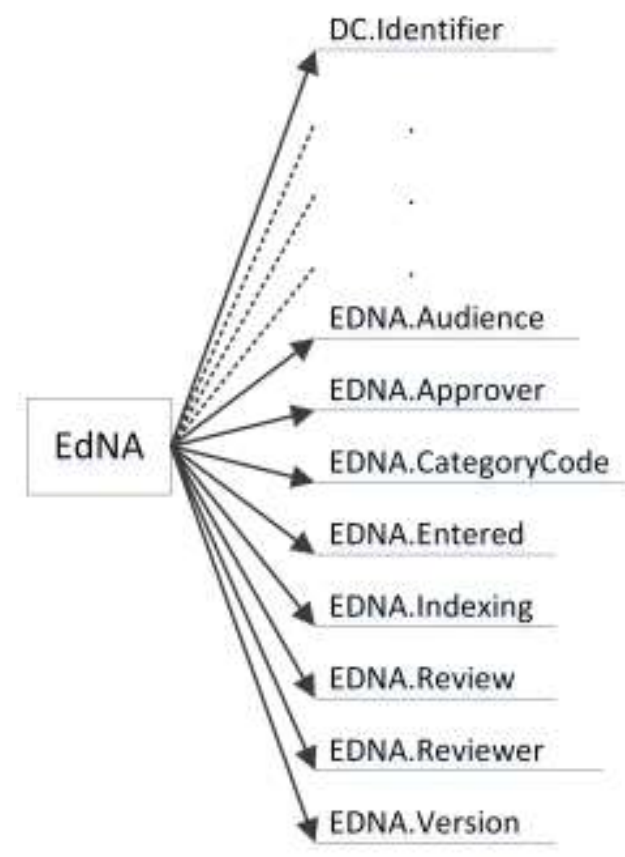

Fig. 2: Metadatos EdNA.

\section{IEEE Learning Object Metadata / IMS Learning Resource Metadata Specification (LOM)}

El esquema de metadatos IEEE Learning Object Metadata (LOM) fue creado por el Comité de Estándares de Tecnologías de Aprendizaje del Instituto de Ingenieros Eléctricos y Electrónicos (IEEE LTSC, Institute for Electrical and Electronic Engineers Learning Technology Standards Committee), basándose en esquemas de metadatos anteriores e influenciado por Dublin Core (Kwon y Moon, 2009). El objetivo de LOM es permitir la descripción estructurada de los recursos educativos, guiar en el proceso de marcación de dichos recursos y así potenciar su búsqueda, evaluación, obtención y utilización (Berlanga y García, 2004) (Fernández-Manjón et al, 2011). LOM se encuentra publicado en los estándares IEEE 1484.12.1 y 1484.12.3. La norma IEEE 1484.12.3, denominada XML Schema Definition Language Binding for LOM, establece cómo representar los metadatos en un formato legible para un computador y se ha centrado en el lenguaje de marcado XML para este fin.

La norma IEEE 1484.12.1, por su parte, define la estructura de los metadatos de LOM de manera jerárquica donde a cada elemento se le asigna una definición, tipo de dato, valores permitidos y si permite multiplicidad o no (Peig, 2003). Los metadatos se clasifican en nueve categorías que corresponden a los niveles superiores de la jerarquía, agrupando aspectos técnicos, educativos, de derechos de autor, ciclo de vida, etc. (Berlanga y García, 2004) (Fernández-Manjón et al, 2011). Cada categoría contiene subcategorías con el fin de dar más detalles sobre la descripción del recurso, por ejemplo, la categoría relacionada con el uso educativo contiene los subelementos: nivel de interactividad, rango de edad típico, tiempo de aprendizaje típico, entre otros (Kwon y Moon, 2009). Las categorías son: General, Ciclo de vida (Lifecycle), MetaMetadatos (Meta-Metadata), Técnico (Technical), Educativo (Educational), Derechos (Rights), Relación (Relation), Anotación (Annotation) y Clasificación (Classification) (ver fig. 3), dentro de las cuales se destacan las categorías Educativo y Clasificación ya que contienen los metadatos que permiten almacenar información de carácter educativo. La categoría Educativo contiene las características pedagógicas del recurso, por ejemplo, tipo de recurso educativo (es una figura, es un ejercicio, etc.), rango típico de edad, etc., y la categoría Clasificación contiene el tema o temas a los que pertenece el recurso (física, historia, literatura, etc.) y se puede establecer con el detalle que se requiera por medio de anidamiento de temas (Fernández-Manjón et al, 2011).

Por otro lado, el área de mayor impacto de IEEE LTSC es la relacionada con los metadatos para la marcación de recursos educativos debido a que LOM es el estándar más utilizado en dicho entorno (CastroGarcía y López-Morteo, 2013) (Rey-López, 2009) (Berlanga y García, 2004) (Fernández-Manjón et al, 2011). A pesar de lo anterior, el trabajo de (Castro-García y López-Morteo, 2013) detalla y clasifica gran cantidad de perfiles y en especial extensiones de LOM realizados por medio de diversas modificaciones, tales como, adición de elementos, exclusión de elementos, modificación del estándar (modificación de tipos de datos, nuevas reglas para el uso de los metadatos), entre otros, evidenciando que LOM no cubre con todos los requerimientos de sus usuarios para diferentes contextos educativos. 


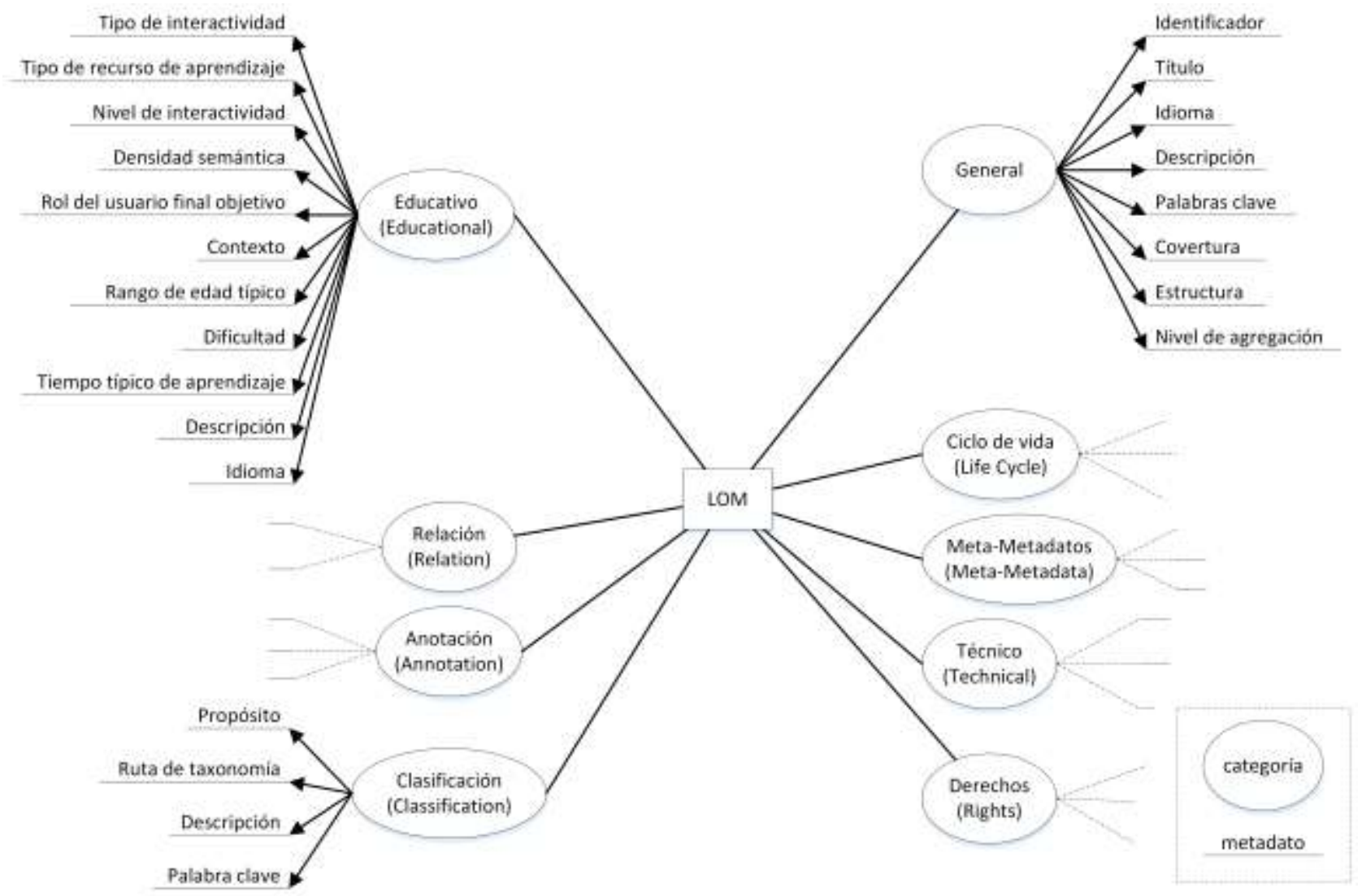

Fig. 3: Estructura de LOM (IMS, 2006).

Es importante conocer la historia de la creación de LOM con el fin de aclarar su nombre y relación con otros estándares. En 1997 el Proyecto IMS (Instructional Management Systems Project) liderado por el consorcio EDUCOM (actualmente EDUCASE) fijó sus esfuerzos en el desarrollo de estándares abiertos para el aprendizaje en línea, incluyendo los metadatos para describir el material educativo. En el mismo año, el Instituto Nacional de Estándares y Tecnología (NIST, National Institute for Standards and Technology) y el grupo de estudio IEEE P.1484 (actualmente el Comité para los Estándares de la Tecnología del Aprendizaje (LTSC, IEEE Learning Technology Standards Committee)) empezaron esfuerzos similares. Los proyectos de EDUCOM y NIST se fusionaron bajo el nombre del Proyecto IMS e iniciaron a trabajar junto con el proyecto europeo ARIADNE. Como fruto de este trabajo conjunto, en 1998 IMS y ARIADNE le presentaron a IEEE una propuesta lo suficientemente sólida para convertirse en la base del borrador de LOM. En 1999, el Proyecto IMS formó el Consorcio Global de Aprendizaje IMS (IMS/GLC, IMS Global Learning Consortium) y ese mismo año dio a conocer la versión 1.0 de la Especificación para Metadatos de Recursos Educativos (Learning Resource Meta-data Specification) la cual obtuvo diferentes revisiones basadas en las actualizaciones de LOM que fueron publicadas hasta el 2001 con la versión 1.2.2 complementadas con una guía de mejores prácticas e implementación y representación de los metadatos por medio de XML, producida por IMS/GCL. En junio de 2002, LOM fue aprobado como estándar IEEE con la referencia IEEE 1484.12.1 - 2002 IEEE LOM Standard for Learning Object Metadata. Debido a que IEEE 1484.12.1 solo especifica los aspectos teóricos del esquema sin incluir información acerca de cómo representar, transmitir y procesar los metadatos, en el 2005, IEEE publicó el estándar IEEE 1484.12.3 - 2005 XML Schema Definition Language Binding for LOM con el fin de proporcionar los mecanismos necesarios para que LOM sea utilizado en la práctica. Debido a que el estándar publicado en 2002 por IEEE se basó en el borrador 6.4 de LOM y la versión 1.2.2 de la especificación de IMS se basó en el borrador 6.1 de LOM, IMS reajustó su trabajo de acuerdo a los estándares de IEEE dando lugar a su versión 1.3. De acuerdo a lo anterior, tanto IEEE LOM e IMS Learning Resource Meta-Data son equivalentes y es posible utilizar el término LOM para cualquiera de los dos (IMS, 2006) (CETIS, 2005).

\section{Learning Resource Metadata Initiative (LRMI)}

La especificación Iniciativa de Metadatos para Recursos Educativos (LRMI, Learning Resource Metadata Initiative) fue desarrollada por las organizaciones Association of Educational Publishers (AEP) y Creative Commons con financiamiento de las fundaciones Bill \& Melinda Gates Fundation y The William and Flora Hewlett Foundation. Es un esquema de marcación sencillo destinado a estandarizar la manera de describir o etiquetar las características educativas de los recursos en la web (Creative Commons, 2013) (DCMI, 
2014) y un paso clave para el desarrollo de una mejor experiencia de búsqueda para los educadores y estudiantes. Además, algunas de sus características permitirán el desarrollo de sistemas de aprendizaje de próxima generación basados en aprendizaje guiado personalizado (Creative Commons, 2013).

El desarrollo de LRMI se inició en el 2011 y fue inspirado por el surgimiento de Schema.org el cual es un proyecto entre los principales motores de búsqueda Bing, Google, Yahoo y Yandex que busca crear un estándar de marcación de contenidos en la web. AEP y Creative Commons se propusieron extender a Schema.org por medio de un pequeño conjunto de metadatos que permitieran describir la intención educativa de una página web, recurso o pieza de contenido, el resultado de esta propuesta fue la especificación LRMI (LRMI, 2014). En abril de 2013, la versión 1.1 de esta especificación fue aceptada y publicada como una extensión oficial de Schema.org, en consecuencia, quien desee publicar contenido educativo puede utilizar la marcación LRMI para proporcionar metadatos educativos relacionados con sus recursos y tener la seguridad de que dichos metadatos serán reconocidos por los principales motores de búsqueda (Creative Commons, 2013) (DCMI, 2014). En el 2014, la administración de LRMI fue transferida de AEP y Creative Commons a DCMI (Dublin Core Metadata Initiative).

Con el objetivo de interoperar con otras especificaciones, LRMI se basó en diferentes proyectos similares y obtuvo apoyo de sus creadores: IEEE Learning Object Metadata, Dublin Core Metadata Initiative, IMS Global Learning Consortium Learning Resource Metadata, ARIADNE Educational Metadata Recommendation, SCORM, ISO/IEC Metadata for Learning Resources. LRMI extiende a Scheme.org por medio de dos tipos de metadatos educativos, los que contienen las características educativas del recurso (uso educativo, rango típico de edad, tipo de interactividad, tipo de recurso de aprendizaje, etc.), y los que relacionan el recurso con un proceso educativo con el objetivo de permitir su búsqueda en un escenario específico, donde dicho escenario se refiere a un marco educativo, es decir, una descripción estructurada de conceptos educativos como por ejemplo, un plan de estudios, un programa, un conjunto de objetivos formativos, etc. La fig. 4 muestra los dos tipos de metadatos definidos por LRMI versión 1.1 (el primer tipo extiende del elemento CreativeWork y el segundo tipo de AligmentObject) y su relación con los elementos de Schema.org (DCMI, 2014).

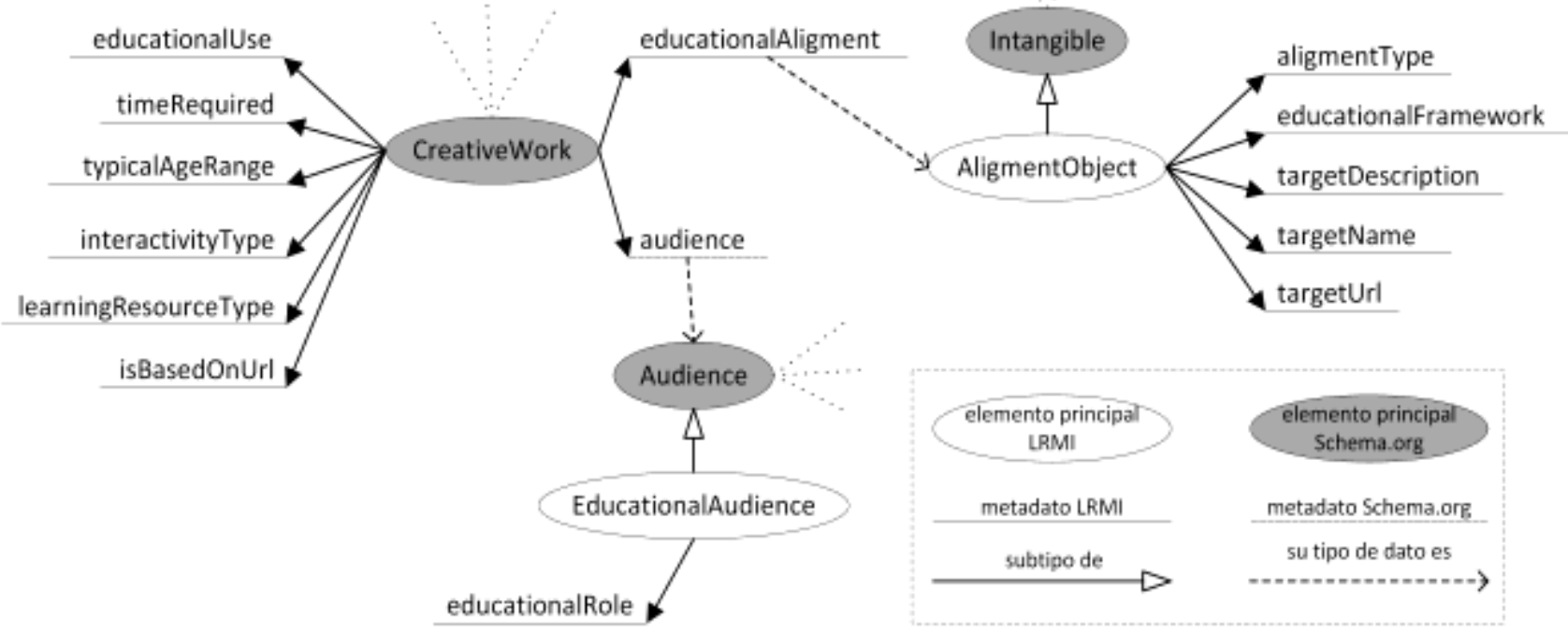

Fig. 4: Elementos definidos por LRMI relacionados con los elementos definidos por Scheme.org.

\section{Metadata for Learning Resources (MLR)}

El esquema de metadatos Metadata for Learning Resources (MLR) fue diseñado por el subcomité SC 36 del comité técnico JTC 1 de la organización ISO/IEC (International Organization for Standardization/International Electrotechnical Commission), y nació con el fin de crear un nuevo esquema útil, adaptable, interoperable y compatible con estándares de metadatos anteriores, por esta razón se basó en las experiencias obtenidas al implementar diferentes normas aceptadas, en especial LOM, y en metadatos generales ampliamente adoptados como los que contiene Dublin Core (Ponds et al, 2011) (Nevile, 2013). Su objetivo principal es facilitar tanto la descripción de los recursos educativos por medio de normas que identifican y especifican los metadatos requeridos para este fin, como la búsqueda, descubrimiento, adquisición, evaluación y uso de estos recursos por parte de los estudiantes, instructores o procesos software automatizados (ISO/IEC, 2011a). 
MLR se encuentra descrito en el estándar ISO/IEC 19788 y se compone de varios módulos. Actualmente se han publicado las primeras cinco partes del estándar. La primera parte ISO/IEC 19788-1 es un marco de definición que contiene los principios y reglas generales que utiliza el estándar para la descripción de un recurso educativo (ISO/IEC, 2011a). La segunda parte ISO/IEC 19788-2 proporciona un conjunto de metadatos básicos para la descripción de un recurso educativo, con base en el estándar Dublin Core (ISO/IEC, 2011b). La tercera parte ISO/IEC 19788-3 proporciona un perfil de aplicación básico como un método recomendado para mejorar la interoperabilidad y como punto de partida para la adopción del estándar (ISO/IEC, 2011c). La cuarta parte ISO/IEC 19788-4 define el conjunto de metadatos de MLR que permiten describir las características técnicas que afectan o limitan la manera en que debe ser utilizado un recurso educativo (ISO/IEC, 2013d). Y la quinta parte ISO/IEC 19788-5 define el conjunto de metadatos de MLR que permiten describir los aspectos educativos del recurso (Ponds et al, 2011).

El módulo ISO/IEC 19788-6 se eliminó del estándar y actualmente, existen cinco partes en desarrollo, las cuales son, ISO/IEC 19788-7 que describe cómo implementar a MLR por medio de un lenguaje comprensible por un computador (XML, RDF, etc.). ISO/IEC 19788-8 que define los metadatos para la descripción de los registros MLR para permitir el seguimiento del proceso de edición de la descripción del recurso educativo (ISO/IEC, 2014e). ISO/IEC 19788-9 que proporciona los metadatos para la descripción de las personas (naturales o jurídicas) que están relacionas con la descripción del recurso educativo (ISO/IEC, 2014f). ISO/IEC 19788-10, la cual es un perfil de aplicación para elementos de acceso, distribución y propiedad intelectual. Finalmente ISO/IEC 19788-11 que indica la migración de LOM a MLR.

La fig. 5 muestra los elementos educativos que componen el módulo ISO/IEC 19788-5 y cómo se relacionan entre sí. Los metadatos se agrupan en clases de acuerdo a su objetivo y cada clase se compone de metadatos $u$ otras clases de metadatos. Las clases y metadatos principales de para describir un recurso educativo son Método educativo, Anotación, Contribuidor, Rol del contribuidor, Audiencia, Nivel de la audiencia, Edad mínima y máxima, Idioma de la audiencia, Rol de la audiencia, Currículo, Tema del currículo, Nivel del currículo y Actividad educativa (Ponds et al, 2011).

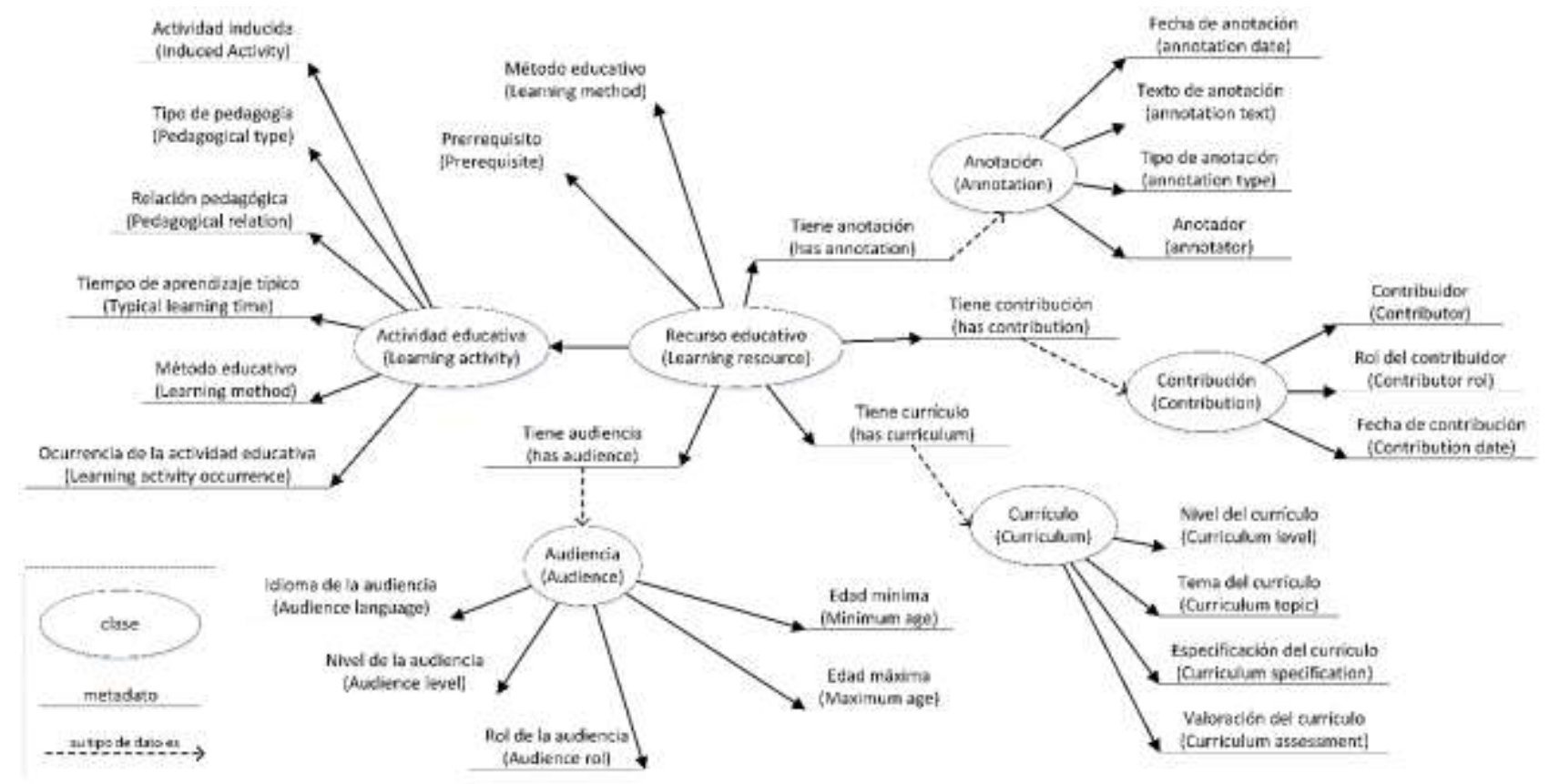

Fig. 5: Estructura de ISO/IEC 19788-5 (Nevile, 2013).

\section{Dublin Core Education Application Profile (DC-Ed AP)}

El perfil de aplicación para la educación de Dublin Core (DC-Ed AP, Dublin Core Education Application Profile) está siendo desarrollado por DC-Education Application Profile Task Force de DCMI desde hace varios años. Su objetivo es definir metadatos que puedan ser utilizados en la descripción de las propiedades educativas de un recurso, además de sugerir el vocabulario adecuado para establecer los valores de los mismos (DCMI, s.f). Durante la revisión de este esquema de metadatos, se evidenció el trabajo que ha realizado la comunidad encargada de su desarrollo, sin embargo, sus participantes son, al mismo tiempo, colaboradores en diferentes proyectos relacionados con educación (IEEE LOM, IMS, MLR, entre otros), lo que ha disminuido su apoyo en el trabajo directo con DC-Ed AP y en consecuencia este proyecto está sin concluir. 
La fig. 6 muestra el alcance que pretende DC-Ed AP, limitándose a la definición de elementos que permitan describir las características educativas del recurso y dejando por fuera de su ámbito de aplicación a los metadatos de descripción general o a los elementos que describen el uso del recurso en contextos diferentes a la enseñanza y el aprendizaje.

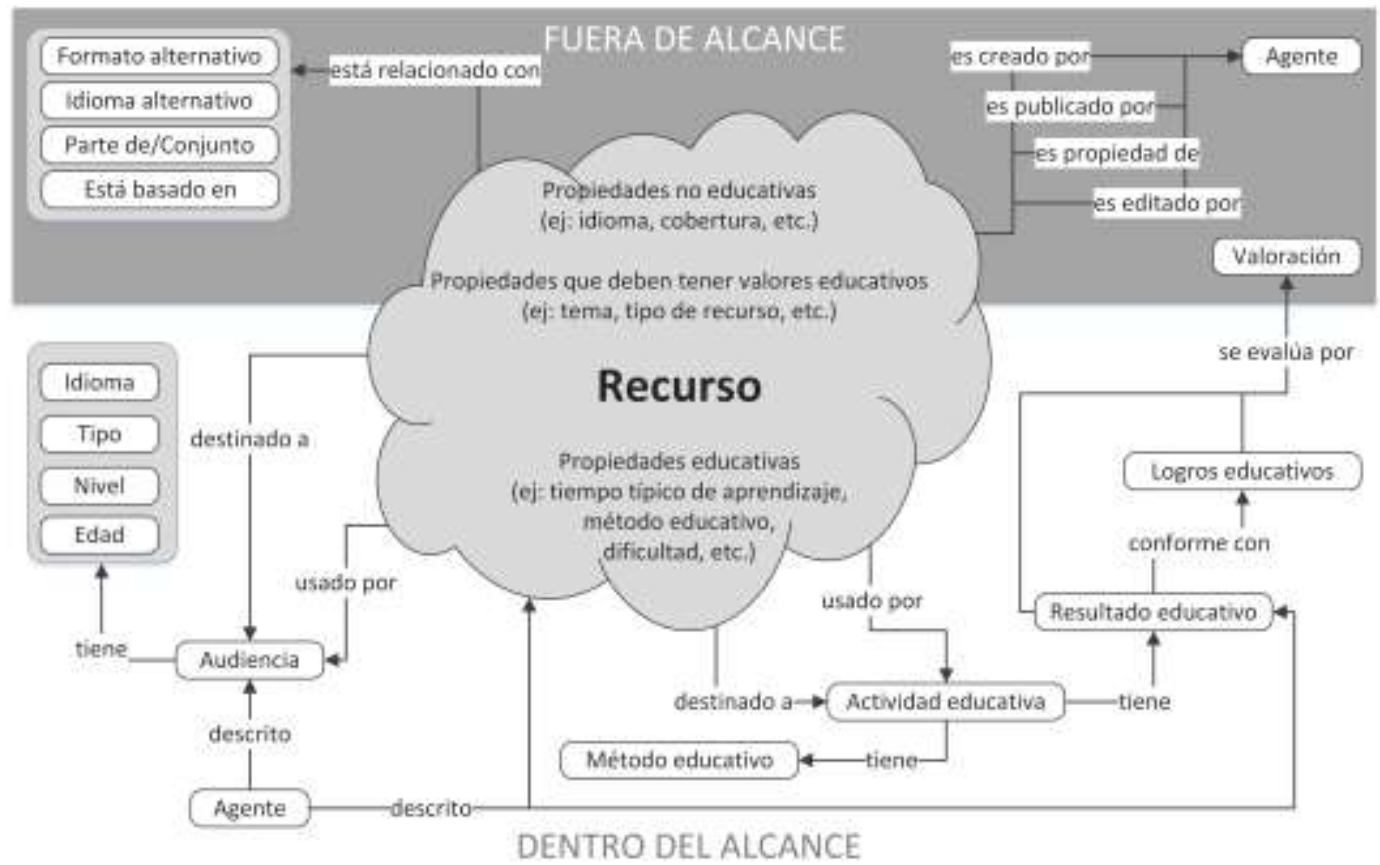

Fig. 6: Alcance de DC-Ed AP (DCMI, 2009)

\section{RELACIÓN ENTRE ESQUEMAS}

A partir del estudio anterior se ha generado la fig. 7 con el fin de mostrar gráficamente las relaciones que existen actualmente entre los esquemas de metadatos para contenidos educativos estudiados.

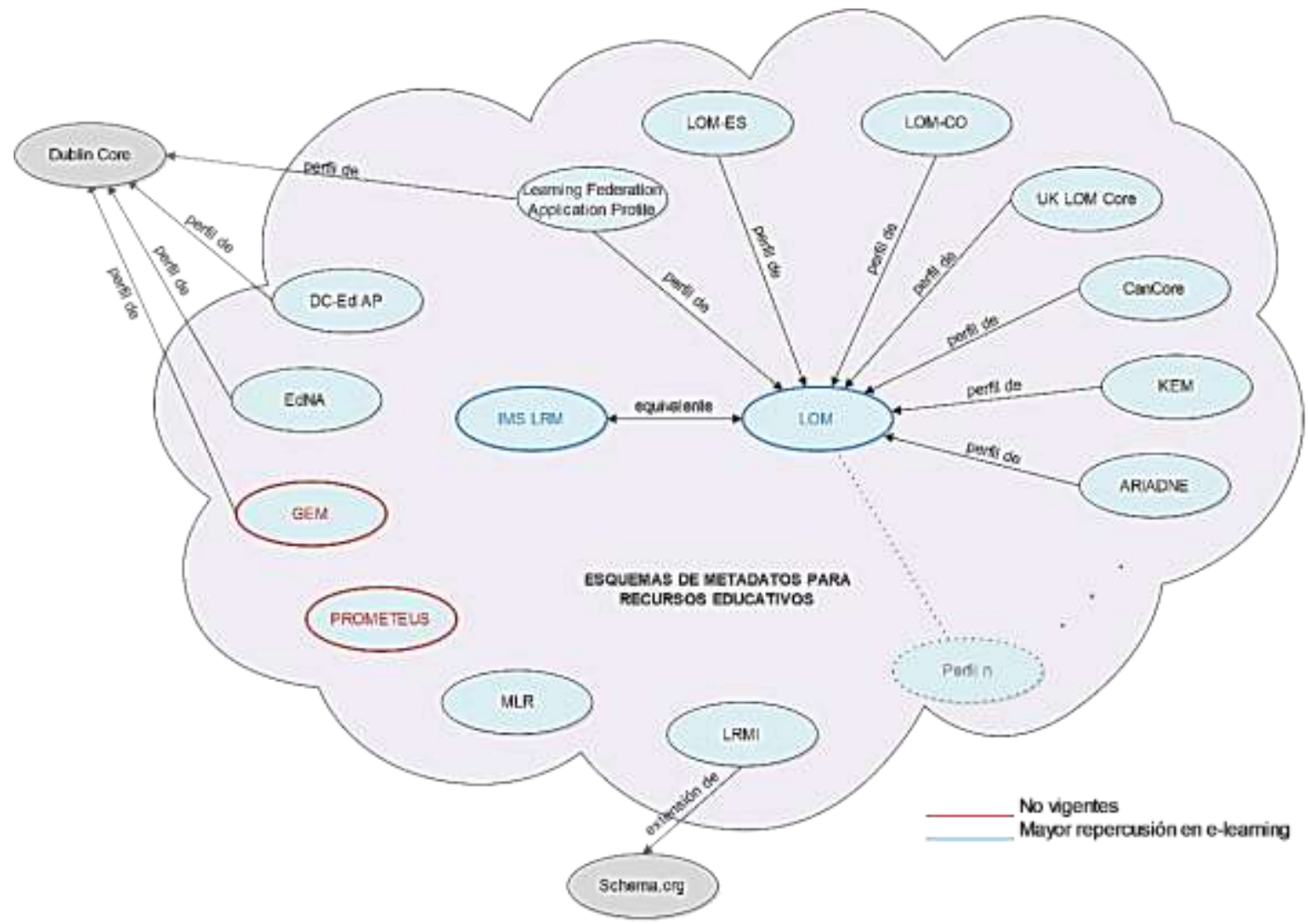

Fig. 7: Relación entre esquemas de metadatos para contenidos educativos 
La tabla 1 muestra una comparación entre los anteriores esquemas de metadatos considerando siete características principales. En primer lugar, se presenta a su desarrollador, es decir, las organizaciones que han creado el esquema. En segundo lugar se proporciona el enlace donde se puede encontrar la información del esquema. En tercer lugar se especifica el nombre del estándar o especificación (si están establecidos). En cuarto lugar, se muestra el esquema de metadatos del cual es una extensión o perfil, es decir, cuál es su esquema base. En quinto lugar se presentan los perfiles y extensiones de cada esquema de metadatos. En sexto lugar se indica si el esquema contiene metadatos que permitan describir las características educativas de un recurso, por ejemplo: rango de edad de la audiencia objetivo, tiempo de aprendizaje aproximado, tipo de recurso educativo (diagrama, presentación, etc.), entre otros. En séptimo y último lugar, se indica si el esquema contiene metadatos que permitan relacionar un recurso con un proceso educativo, por ejemplo: con un currículo o con un plan de estudio.

Tabla 1: Esquemas de metadatos para contenidos educativos.

\begin{tabular}{|c|c|c|c|c|c|c|c|}
\hline $\begin{array}{l}0 \\
\text { है } \\
\text { हे }\end{array}$ & $\begin{array}{l}\frac{0}{0} \\
\frac{\pi}{1} \\
\frac{1}{0} \\
\frac{1}{0} \\
\infty \\
0 \\
0 \\
0\end{array}$ & $\begin{array}{l}0 \\
\frac{\mathscr{T}}{\pi} \\
\mathbb{U}\end{array}$ & 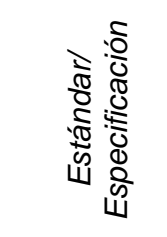 & 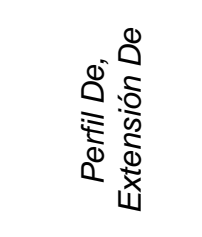 & 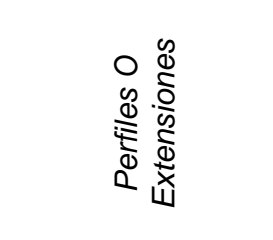 & 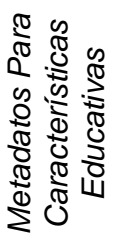 & 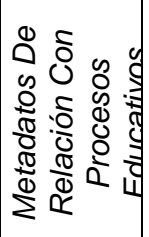 \\
\hline EdNA & $\begin{array}{l}\text { EdNA (Education } \\
\text { Network } \\
\text { Australia) }\end{array}$ & $\begin{array}{l}\text { http://edna.wikis } \\
\text { paces.com/ }\end{array}$ & No aplica & $\begin{array}{l}\text { PERFIL DE: } \\
\text { Dublin Core }\end{array}$ & $\begin{array}{l}\text { PERFILES: } \\
\text { Edna metadata } \\
\text { application profile } \\
2.0\end{array}$ & No & No \\
\hline LOM & $\begin{array}{l}\text { Learning } \\
\text { Technology } \\
\text { Standards } \\
\text { Committee LTSC } \\
\text { de IEEE }\end{array}$ & $\begin{array}{l}\text { http://standards.i } \\
\text { eee.org/ }\end{array}$ & $\begin{array}{l}\text { IEEE } \\
1484.12 .1 \\
y \\
1484.12 .3\end{array}$ & No aplica & $\begin{array}{l}\text { PERFILES: } \\
\text { - LOM-ES } \\
\text { (España) } \\
\text { - LOM-CO } \\
\text { (Colombia) } \\
\text { - UK LOM Core } \\
\text { (Reino Unido) } \\
\text { - CanCore } \\
\text { (Canadian Core } \\
\text { Learning } \\
\text { Resource } \\
\text { Metadata } \\
\text { Protocol) } \\
\text { - KEM } \\
\text { - ARIADNE } \\
\text { - Learning } \\
\text { Federation } \\
\text { Metadata } \\
\text { Application } \\
\text { Profile. } \\
\text { - SingCore } \\
\end{array}$ & Sí & No \\
\hline LRMI & $\begin{array}{l}\text { Association of } \\
\text { Educational } \\
\text { Publishers (AEP) } \\
\text { y Creative } \\
\text { Commons } \\
\end{array}$ & $\begin{array}{l}\text { http://www.Irmi.n } \\
\text { et/ }\end{array}$ & No aplica & $\begin{array}{l}\text { EXTENSIÓN } \\
\text { DE: } \\
\text { Schema.org }\end{array}$ & No aplica & Sí & Sí \\
\hline MLR & ISO/IEC & $\begin{array}{l}\text { http://www.iso.or } \\
\text { g/ }\end{array}$ & $\begin{array}{l}\text { ISO/IEC } \\
19788\end{array}$ & No aplica & No aplica & Sí & Sí \\
\hline $\begin{array}{l}\text { DC-Ed } \\
\text { AP }\end{array}$ & $\begin{array}{l}\text { Dublin Core } \\
\text { Metadata } \\
\text { Initiative DCMI }\end{array}$ & $\begin{array}{l}\text { http://dublincore. } \\
\text { org/ }\end{array}$ & No aplica & $\begin{array}{l}\text { PERFIL DE: } \\
\text { Dublin Core }\end{array}$ & No aplica & $\begin{array}{l}\text { Sin } \\
\text { publi } \\
\text { car }\end{array}$ & $\begin{array}{l}\text { Sin } \\
\text { publi } \\
\text { car }\end{array}$ \\
\hline
\end{tabular}

A partir del análisis de los esquemas de metadatos realizado y presentado en la fig. 7 y la tabla 1, se pueden resaltar varios aspectos. Es importante aclarar que Dublin Core es uno de los primeros estándares de metadatos para describir recursos digitales (Weibel et al, 1998) que ha tenido una amplia difusión en el mundo, y aunque su objetivo no es la marcación de recursos educativos, se ha convertido en la base de muchos esquemas que sí tienen este propósito, como muestra se encuentran sus perfiles de aplicación: EdNA, DC-Ed AP, Learning Federation Application Profile (también perfil de LOM) y GEM (no está vigente debido a la falta de financiación). De igual manera, Dublin Core ha sido el estándar en el cual se fundamentan otros esquemas de metadatos para contextos diferentes al educativo. Lo anterior refleja la preferencia de algunos autores de optar por la simpleza y los metadatos mínimos necesarios para describir un recurso. 
Por otro lado, algunos estudios sostienen que los proyectos que implementan LOM solamente utilizan entre el $50 \%$ y $60 \%$ de sus elementos, además de que el uso de sus metadatos educativos es bajo y la relación entre el nombre de los elementos y sus valores es imprecisa (Ponds et al, 2011) (Castro-García y LópezMorteo, 2013), como prueba de lo anterior se evidencia el gran número de extensiones y perfiles de aplicación del estándar, los cuales disminuyen los problemas anteriores y abarcan un mayor número de requisitos de los usuarios. Sin embargo, el surgimiento de dichos perfiles y extensiones se podría interpretar, por una parte, como el gran interés de la comunidad académica hacia el desarrollo de esquemas de metadatos para contenidos educativos utilizando LOM, y por otra parte, como la incapacidad de LOM para satisfacer todas las necesidades de los usuarios en diferentes contextos educativos, razón por la cual debe ser modificado (Castro-García y López-Morteo, 2013) (Solomou et al, 2015). A pesar de lo anterior, LOM es el esquema de metadatos para contenidos educativos más utilizado e influyente en los procesos de aprendizaje soportados por las TIC.

Los estándares más recientes MLR y LRMI, a diferencia de LOM y otras normas más antiguas, pretenden ofrecer un mayor número de elementos pedagógicos siendo más claros en su definición con el fin de mejorar la relación entre el metadato y su valor (Ponds et al, 2011), además, agregan elementos que permiten asociar el recurso con un proceso educativo establecido. En cuanto a LRMI, es importante resaltar que al ser una extensión de Schema.org, permitirá que los recursos marcados con su modelo sean reconocidos y encontrados por los principales motores de búsqueda. MLR, por su parte, no tiene una relación directa con algún esquema, pero sí ha tenido en cuenta diferentes trabajos para su desarrollo, entre ellos LOM.

En representación de otros esfuerzos con el mismo enfoque está DC-Ed AP que ha sido desarrollado con la colaboración de grupos de estandarización relacionados con metadatos educativos (IEEE LOM, IMS, ISO $M L R$ ), reflejando la necesidad de diferentes organizaciones de tener un modelo de metadatos para este tipo de contenido, pero la inexistencia de un estándar interoperable con sus modelos base ya establecidos, hace necesaria la creación de un perfil o extensión compatible con sus normas y que al mismo tiempo tome como referencia lo establecido por proyectos relacionados. Finalmente, EdNA refleja la simplicidad por la que optan muchas normas y por lo tanto sus metadatos no representan todas las características educativas de un recurso, sin embargo, esto no impide su uso en contextos de enseñanza y aprendizaje.

\section{CONCLUSIONES}

En este trabajo se ha realizado un estudio y análisis exhaustivo de los esquemas de metadatos vigentes más conocidos y utilizados para la descripción de recursos educativos. El resultado de este análisis se refleja en un gráfico de relación entre los esquemas (perfil de, extensión de, equivalente a) y en una tabla comparativa de las características más importantes de cada uno de ellos: desarrollador, enlace, estándar o especificación, esquema de metadatos base, perfiles y extensiones, contiene metadatos que permiten describir las características educativas de un recurso, contiene metadatos que permiten relacionar un recurso con un proceso educativo. Entre las conclusiones más relevantes de este análisis se destacan:

LOM es el estándar de metadatos con mayor repercusión en e-learning y continua vigente (FernándezManjón et al, 2011) (Al-Khalifa et al, 2006), pero la gran cantidad de perfiles de aplicación que se han implementado a partir de él evidencian su incapacidad de soportar la necesidades de diferentes contextos educativos. En cuanto a su denominación, LOM es equivalente a IMS LRM, por lo que es correcto referirse a él con cualquiera de estos dos nombres.

Los esquemas de metadatos más actuales (MLR y LRMI) son conscientes de la falta de elementos pedagógicos de los esquemas más antiguos y por esta razón definen metadatos que satisfacen esta necesidad (por ejemplo: uso educativo, tipo de pedagogía, método educativo, entre otros). Otro aspecto que estos esquemas abarcan, es la descripción de la relación del recurso con un proceso educativo (un currículo, un plan de estudio, un conjunto de objetivos, etc.).

\section{REFERENCIAS}

Al-Khalifa, H. S., Davis, H. C. The evolution of metadata from standards to semantics in E-learning applications. In Proceedings of the seventeenth conference on Hypertext and hypermedia. Pag. 69-72, (2006)

Barker, P., Campbell, L., Metadata for Learning Materials: An Overview of Existing Standards and Current Developments. Technology, Instruction, Cognition and Learning. Vol 7 (3-4), pp 225- 243, (2010) 
Berlanga A., García F.J., Introducción a los Estándares y Especificaciones para Ambientes e-learning. In F.J. Garcia and M.N. Moreno (Eds.), Proceedings of Tendencias en el Desarrollo de Aplicaciones Web. Jornadas Desarrollo Web (Salamanca, Spain, November 20-27), Ediciones Universidad de Salamanca. Pag 25-37, (2004)

Castro-García L., López-Morteo G., An international analysis of the extensions to the IEEE LOMv1.0 metadata standard. doi:10.1016/j.csi.2013.04.006, Computer Standards \& Interfaces, Volume 35, Issue 6, Pag 567-581, (2013)

CETIS - JISC's Centre For Educational Technology Interoperability Standards, What is IEEE Learning Object Metadata / IMS Learning Resource Metadata?, Reporte técnico, (2005)

Creative Commons, LRMI Accepted into Schema.org, (en la web:

https://blog.creativecommons.org/2013/05/05//rmi-accepted-into-schema-org/, acceso: 1 de marzo de 2016), Creative Commons News, (2013)

DCMI - DC-Education Application Profile Task Group of Dublin Core Metadata Iniciative. Dublin Core education application profile (Working Draft of v0.4), (en la web: https://docs.google.com/document/d/15K2KBrxOLPGkfnCa17gowPRReC-43DYRiSGfJLkjx8w/edit, acceso: 19 de enero de 2015), DCMI (s.f)

DCMI - Dublin Core Metadata Iniciative, DC-Education, [figura], (en la web: http://dublincore.org/moinmoinwiki-archive/educationwiki/attachments/DC-Education, acceso: 5 de febrero de 2015). DCMI (2009)

DCMI - Dublin Core Metadata Iniciative, LRMI Version 1.1, (en la web: http://dublincore.org/dcx/lrmiterms/1.1/, acceso: 3 de febrero de 2015). LRMI Metadata Terms, October (2014)

EdNA Metadata Standard Working Group, EdNA Metadata Standard 1.1, (en la web: http://edna.wikispaces.com/, acceso: 23 de enero de 2015). EdNA (s.f)

Fernández-Manjón B., Moreno-Ger P., Sierra-Rodríguez J. L., Martínez-Ortíz I., Uso de estándares aplicados a TIC en educación, (en la web:

http://books.google.es/books?id=MBEbAgAAQBAJ\&printsec=frontcover\&source=gbs_ge_summary_r\&cad= 0 - $v=$ =nepage\&q\&f=false, acceso: 18 de marzo de 2015) Ministerio de Educación de España, España, (2011)

Frederick D.E, 1 - Understanding eBooks, metadata, and managing metadata, doi:10.1016/B978-0-08100151-6.00001-9. In Managing Ebook Metadata in Academic Libraries, Chandos Publishing, pp 1-10, Boston, USA (2016).

Friesen, N. Three objections to learning objects and e-learning standards. Online education using learning objects. McGreal, R. (Ed.). London, Pag. 59-70, (2004).

Hoel, T. Mason, J. Expanding the Scope of Metadata and the Issue of Quality. In Proceedings of the 19th International Conference on Computers in Education. Asia-Pacific Society for Computers in Education. Chiang Mai, Thailand, (2011)

IMS Global Learning Consortium, Inc., IMS Meta-data Best Practice Guide for IEEE 1484.12.1-2002 Standard for Learning Object Metadata - Version 1.3 Final Specification, Reporte Técnico, (2006)

ISO/IEC, ISO/IEC 19788-1 Information technology - Learning, education and training - Metadata for learning resources - Part 1: Framework, International Standard, (2011)

ISO/IEC, ISO/IEC DIS 19788-2 Information technology — Learning, education and training - Metadata for learning resources - Part 2: Dublin Core elements, Draft International Standard, (2011)

ISO/IEC, ISO/IEC DIS 19788-3 Information technology — Learning, education and training - Metadata for learning resources - Part 3: Basic application profile, Draft International Standard, (2011)

ISO/IEC, ISO/IEC DIS 19788-4 Information technology - Learning, education and training - Metadata for learning resources - Part 4: Technical elements, Draft International Standard, (2013) 
ISO/IEC, ISO/IEC DIS 19788-8 Information technology - Learning, education and training - Metadata for learning resources —Part 8: Data elements for MLR records, Draft International Standard, (2014)

ISO/IEC, ISO/IEC DIS 19788-9 Information technology - Learning, education and training - Metadata for learning resources - Part 9: Data elements for Persons, Draft International Standard, (2014)

Kwon B.-I., Moon N.-M. A study of metadata design for e-Learning marketplace based on IPTV. doi:10.1145/1644993.1645008, In Proceedings of the 2009 International Conference on Hybrid Information Technology (ICHIT '09). Pag 79-85. ACM, New York, NY, USA, (2009)

Learning Resource Metadata Initiative (LRMI), LRMI Transfers Stewardship, (en la web: http://www.Irmi.net//rmi-transfers-stewardship, acceso: 2 de febrero de 2015). LRMI (2014)

Morales, E. M., Gestión del conocimiento en sistemas e-learning, basado en objetos de aprendizaje, cualitativa y pedagógicamente definidos, Tesis doctoral, Universidad de Salamanca, España, (2007)

Nevile, L. DC Metadata is Alive and Well (and has Influenced a New Standard for Education). International Conference on Dublin Core and Metadata Applications, North America, (2013)

N. I. S. O. NISO. Understanding metadata, Technical report, National Information Standards Organization NISO, (2004).

Peig E., Interoperabilidad de Metadatos en sistema\}+

,erdfas distribuidos, Tesis doctoral, Universitat Pompeu Fabra, (2003)

Pons, D., Hilera, J.R., Pagés, C. (2011). ISO/IEC 19788 MLR: Un Nuevo Estándar de Metadatos para Recursos Educativos. IEEE-RITA, ISSN: 2255-5706. (en línea; http://dblp.uni-trier.de/db/journals/ieeerita/ieee-rita6.html\#BetrianHP11, acceso 25 de marzo 2015). Vol. 6, Núm. 3, Pag. 140-145, (2011)

Rey-López M., Marco Conceptual y Arquitectura para el Aprendizaje Personalizado a través de Televisión Digital Interactiva, Tesis doctoral, Universidad de Vigo, España, (2009)

Solomou, G., Pierrakeas, C., \& Kameas, A. Characterization of educational resources in e-learning systems using an educational metadata profile. Journal of Educational Technology \& Society, 18(4), Pag. 246-260, (2015).

Waters, J. K. Sifting the Data: The Learning Resource Metadata Initiative Has a Complicated Name but a Simple Purpose: To Make Web Searches More Useful for Students and Teachers. THE Journal (Technological Horizons In Education). Vol 40, Issue 1, (2013)

Weibel, S., Kunze, J., Lagoze, C., Wolf, M. Dublin Core Metadata for Resource Discovery, IETF Req. Comments, vol. 2413, pp. 1-8, Sep. (1998) 